F. BLANCHARD

B. Host

\title{
A. MAASS
}

\section{Représentation par automate de fonctions continues de tore}

Journal de Théorie des Nombres de Bordeaux, tome 8, no 1 (1996), p. $205-214$

<http://www.numdam.org/item?id=JTNB_1996_8_1_205_0>

(C) Université Bordeaux 1, 1996, tous droits réservés.

L'accès aux archives de la revue « Journal de Théorie des Nombres de Bordeaux » (http://jtnb.cedram.org/) implique l'accord avec les conditions générales d'utilisation (http://www.numdam.org/conditions). Toute utilisation commerciale ou impression systématique est constitutive d'une infraction pénale. Toute copie ou impression de ce fichier doit contenir la présente mention de copyright.

\section{Numdam}

Article numérisé dans le cadre du programme

Numérisation de documents anciens mathématiques

http://www.numdam.org/ 
Journal de Théorie des Nombres

de Bordeaux 8 (1996), 205-214

\title{
Représentation par automate de fonctions continues de tore
}

\author{
par F. BLANCHARD, B. HOST ET A. MAASS
}

\begin{abstract}
RÉSUMÉ. Soient $A_{p}=\{0, \ldots, p-1\}$ et $Z \subseteq A_{p}^{\mathbb{N}} \times A_{p}^{\mathbb{N}}$ un sous-système. $Z$ est une représentation en base $p$ d'une fonction $f$ du tore si pour tout point $x$ du tore, ses développements en base $p$ sont liés par le couplage $Z$ aux développements en base $p$ de $f(x)$. On prouve que si $f$ est représentable en base $p$ alors $f(x)=\left(u x+\frac{m}{p-1}\right) \bmod 1$, où $u \in \mathbb{Z}$ et $m \in A_{p}$. Réciproquement, toutes les fonctions de ce type sont représentables en base $p$ par un transducteur. On montre finalement que les fonctions du tore qui peuvent être représentées par automate cellulaire sont exclusivement les multiplications par un diviseur d'une puissance de la base.
\end{abstract}

\section{Introduction.}

La représentation des fonctions continues de l'intervalle par des systèmes discrets simples, par exemple les automates cellulaires, les automates finis, les transducteurs, a fait l'objet de plusieurs travaux récents; voir par exemple $[\mathrm{M}],[\mathrm{BG}]$ ou $[\mathrm{BDT}]$. Les motivations viennent d'une part de l'informatique théorique, notamment du calcul arithmétique en ligne, et d'autre part de la théorie ergodique.

Soit $p$ un entier positif, et notons $A_{p}=\{0, \ldots, p-1\}$. A chaque élément $x$ du tore est associé l'ensemble de ses développements en base $p, T_{p}(x) \subseteq A_{p}^{\mathbb{N}}$. Tout le monde sait que $\left|T_{p}(x)\right| \leq 2$. Soit $Z \subseteq A_{p}^{\mathbb{N}} \times A_{p}^{\mathbb{N}}$ un couplage entre $A_{p}^{\mathbb{N}}$ et un sous-système $X_{S} \subseteq A_{p}^{\mathbb{N}}$. $Z$ représente une fonction $f$ du tore si pour chaque $\underline{x} \in T_{p}(x)$ les $\underline{y} \in A_{p}^{\mathbb{N}}$ tels que $(\underline{x}, \underline{y}) \in Z$ représentent le même élément $y=f(x)$ du tore. Un tel couplage est appellé une représentation en base $p$ de $f$. La proposition 2.2 établit que les fonctions ayant une représentation en base $p$ sont continues. II faut remarquer qu'à toute fonction $f$ du tore est associé un sous-ensemble $Z$ de $A_{p}^{\mathbb{N}} \times A_{p}^{\mathbb{N}}$ qui la représente. $\Pi$ suffit de prendre $Z=\left\{(\underline{x}, \underline{y}) \in A_{p}^{\mathbb{N}} \times A_{p}^{\mathbb{N}} / \underline{x}\right.$ est un développement de $x$ et $\underline{y}$ de $f(x)$ \}. Si $f$ est continue, $Z$ est fermé, mais pas nécessairement invariant par le décalage.

Manuscrit reçu le 25 novembre 1993 
Dans cette article on s'intéresse en particulier aux couplages $Z$ engendrés par automates cellulaires et transducteurs. Dans le premier cas $Z$ est simplement le graphe d'une application.

La partie 2 est consacrée aux définitions et résultats préliminaires.

L'objet essentiel de la troisième partie est de démontrer que trois familles de fonctions sont identiques : l'une, les fonctions représentables, est définie en termes de couplages dynamiques; la seconde, les fonctions représentables par transducteur, est définie par automates; et la troisième se décrit de façon purement arithmétique : ce sont les fonctions $f(x)=\left(u x+\frac{m}{p-1}\right)$ $\bmod 1$, où $u \in \mathbb{N}$ et $m \in A_{p}$.

On prouve dans le théorème 3.3 que lorsqu'une fonction du tore $f$ a une représentation en base $p$, elle est de la forme $f(x)=\left(u x+\frac{m}{p-1}\right) \bmod$ 1 , où $u \in \mathbb{N}$ et $m \in A_{p}$; la démonstration repose essentiellement sur le fait peu connu que toute fonction continue du tore, commutant avec la multiplication par $p$, est de cette même forme. On applique ce résultat aux automates cellulaires et transducteurs. II est établi dans [BDT], et rappelé ici, qu'on peut représenter par transducteurs toutes les fonctions $f(x)=$ $\left(u x+\frac{m}{p-1}\right) \bmod 1$. Finalement, le corollaire 3.5 établit que les automates cellulaires ne peuvent représenter que les fonctions du type $f(x)=(u x)$ $\bmod 1$, où $u$ est un entier qui divise une puissance positive de $p$.

Le résultat obtenu - il n'existe pas d'autres fonctions représentables que celles que nous savions déjà l'être au départ - est limitatif : on peut l'interpréter en allant jusqu'à dire que les seules fonctions "bien adaptées" à la représentation en base entière sont celles décrites plus haut. Par contre, on peut introduire une forme plus faible de la représentabilité, par exemple en exigeant que la fonction soit seulement définie par transducteur, surjective et continue en dehors des points $p$-adiques. On définit ainsi une classe bien plus vaste de fonctions, dont on ne sait que peu de choses; toutefois, comme elles sont toutes mesurables et préservent la mesure uniforme, il serait fort intéressant d'étudier la dynamique mesurée qu'elles définissent chacune sur le tore.

Nous remercions B. Weiss de nous avoir signalé la relation avec les résultats figurant dans [JR].

\section{Définitions et Notations.}

Soit $A$ un alphabet fini. On appellera $A_{k}$ l'alphabet $\{0, \ldots, k-1\}$. On note $A^{*}$ l'ensemble des suites finies ou mots de $A$. Un langage est une partie quelconque de $A^{*}$. Dans cet article $\mathbb{N}$ représente l'ensemble des 
entiers positifs. $A^{\mathbb{N}}$ est l'ensemble des suites infinies $\underline{x}=\left(x_{i}\right)_{i \in \mathbb{N}}$ où $x_{i} \in A$. On munit $A^{\mathbb{N}}$ de la topologie produit et du décalage $\sigma: \sigma(\underline{x})=\left(x_{i+1}\right)_{i \in \mathbb{N}}$. Un sous-système $S \subseteq A^{\mathbb{N}}$ est un ensemble fermé et $\sigma$ invariant. Le langage associé au sous-système $S$ est $L(S)=\left\{w=w_{0} \ldots w_{n} \in A^{*} / \exists \underline{x} \in S\right.$ tel que $\left.w=x_{0} \ldots x_{n}\right\}$. I est bien connu que $S$ est complétement décrit par son langage. Un couplage entre deux sous-systèmes $X \subseteq A^{\mathbb{N}}$ et $Y \subseteq B^{\mathbb{N}}$ est un sous-système $Z \subseteq A^{\mathbb{N}} \times B^{\mathbb{N}}$ dont les projections sur les première et deuxième composantes sont $X$ et $Y$ respectivement.

Soit $p \in \mathbb{N}$. On note $\mathbb{T}$ le tore $\mathbb{R} / \mathbb{Z}$ et on pose $[\cdot]_{p}=(\cdot) \bmod p$. A chaque point $x \in \mathbb{T}$ on associe l'ensemble des développements en base $p$,

$$
T_{p}(x)=\left\{\underline{x}=\left(x_{i}\right)_{i \in \mathbb{N}} \in A_{p}^{\mathbb{N}} / x=V_{p}(\underline{x})\right\} .
$$

où $V_{p}(\underline{x})=\left[\sum_{i \in \mathbb{N}} \frac{x_{i}}{p^{i}}\right]_{1}$ est la valuation de $\underline{x} . T_{p}$ est 1-1 sauf pour les éléments p-adiques du tore, $x=\frac{k}{p^{l}}$ avec $l \in \mathbb{N}, k \in\left\{0, \ldots, p^{l}-1\right\}$, pour lesquels $T_{p}(x)=\left\{\underline{x}_{1}=x_{1} \ldots x_{l} 0^{\infty}, \underline{x}_{2}=x_{1} \ldots x_{l-1}\left(x_{l}-1\right)(p-1)^{\infty}\right\}$.

DÉFINITION 2.1. Soit $f: \mathbb{T} \rightarrow \mathbb{T}$ une fonction du tore. On dira que $f$ a une représentation en base $p \in \mathbb{N}$, s'il existe un sous-système $Z_{f} \subseteq A_{p}^{\mathbb{N}} \times A_{p}^{\mathbb{N}}$ tel que : (i) quels que soient $x \in \mathbb{T}, \underline{x} \in T_{p}(x), \underline{y} \in A_{p}^{\mathbb{N}}$ avec $(\underline{x}, \underline{y}) \in Z_{f}$ alors $\underline{y} \in T_{p}(f(x))$ et (ii) si on note $X_{E}$ et $X_{S}$ les projections de $Z_{f}$ sur les première et deuxième composantes respectivement, $X_{E}=A_{p}^{\mathbb{N}}$. La condition (ii) fait de $Z_{f}$ un couplage entre $A_{p}^{\mathbb{N}}$ et $X_{S}$.

Le fait d'être représentable en base $p$ est une propriété forte. Un couplage qui représente une fonction doit coupler tous les développements (il y en a au plus deux) d'un point du tore avec des développements de son image. La proposition suivante met en valeur deux propriétés cruciales des fonctions représentables.

Proposition 2.2. Si la fonction $f: \mathbb{T} \rightarrow \mathbb{T}$ est représentable en base $p \in \mathbb{N}$, elle est continue et commute avec la multiplication par $p(\bmod 1)$.

Preuve. La continuité découle directement de la compacité du sous-système $Z_{f}$ qui représente $f$. En effet, fixons $x \in \mathbb{T}$. Soit $\left(x_{n}\right)_{n \in \mathbb{N}} \subseteq \mathbb{T}$ tel que $\lim _{n \rightarrow \infty} x_{n}=x$ et $\lim _{n \rightarrow \infty} f\left(x_{n}\right)=z \in \mathbb{T}$. II est facile de voir qu'il existe $\underline{x}_{n_{k}} \in$ $T_{p}\left(x_{n_{k}}\right), k \in \mathbb{N}$, telle que $\lim _{k \rightarrow \infty} \underline{x}_{n_{k}}=\underline{x} \in T_{p}(x)$. Pour simplifier on va oublier les $n_{k}$. Il découle de 2.1 (ii), qu'il existe $\underline{y}_{n}=\left(y_{i, n}\right)_{i \in \mathbb{N}} \in X_{S}, n \in \mathbb{N}$, tel que $\left(\underline{x}_{n}, \underline{y}_{n}\right) \in Z_{f}$. En outre, via l'extraction d'une sous-suite, on peut 
supposer $\lim _{n \rightarrow \infty} \underline{y}_{n}=\underline{y}=\left(y_{i}\right)_{i \in \mathbb{N}}$. Comme $Z_{f}$ est fermé $(\underline{x}, \underline{y}) \in Z_{f}$. Mais $Z_{f}$ représente $f$, donc

$$
\lim _{n \rightarrow \infty} f\left(x_{n}\right)=\lim _{n \rightarrow \infty} V_{p}\left(\underline{y}_{n}\right)=z=f(x)=V_{p}(\underline{y}) .
$$

En conséquence, si on se donne une suite $\left(x_{n}\right)_{n \in \mathbb{N}} \subseteq \mathbb{T}$ qui converge vers $x$, toute sous-suite $\left(f\left(x_{n_{k}}\right)\right)_{k \in \mathbb{N}}$ converge vers $f(x)$, d'où la continuité de $f$.

La commutation avec $\times p(\bmod 1)$ résulte immédiatement de l'invariance de $Z_{f}$ par $\sigma \times \sigma$.

On s'intéresse ici avant tout aux sous-systèmes $Z_{f}$ engendrés par transducteurs et automates cellulaires. En général ils ne représentent pas une fonction.

DÉFInITION 2.3. Un transducteur est un quadruplet $\mathcal{T}=\left(A_{E}, A_{S}, G, \delta\right)$ où $A_{E}$ et $A_{S}$ sont des alphabets finis d'entrée et sortie, respectivement; $G$ est un graphe orienté fini, ayant $K$ pour ensemble des arcs; et enfin $\delta: K \rightarrow A_{E} \times A_{S}$ est la fonction de transduction : un arc $e \in K$ est étiqueté $\left(a_{E}, a_{S}\right) \in A_{E} \times A_{S}$ si $\delta(e)=\left(a_{E}, a_{S}\right)$. Soient $w_{E}=\left(w_{1}, \ldots, w_{n}\right) \in A_{E}^{*}$ et $w_{S}=\left(w_{1}^{\prime}, \ldots, w_{n}^{\prime}\right) \in A_{S}^{*}$. On dira que $w_{S}$ est une transduction de $w_{E}$, et on notera $w_{S} \in \mathcal{T}\left(w_{E}\right)$, s'il existe un chemin sur le graphe $G, v=e_{1}, \ldots, e_{n}$, tel que $\delta\left(e_{i}\right)=\left(w_{i}, w_{i}^{\prime}\right)$ pour $i=1, \ldots, n$. Le langage associé à $\mathcal{T}$ est $L(\mathcal{T})=\left\{\left(w_{E}, w_{S}\right) \in A_{E}^{*} \times A_{S}^{*} / w_{S} \in \mathcal{T}\left(w_{E}\right)\right\}$.

Au transducteur $\mathcal{T}$ on associe deux couplages. Le premier, qu'on lit de gauche à droite, $Z_{g} \subseteq\left(A_{E} \times A_{S}\right)^{\mathbb{N}}$ tel que $L\left(Z_{g}\right)=L(\mathcal{T})$, et le second, $Z_{d} \subseteq$ $\left(A_{E} \times A_{S}\right)^{\mathbb{N}}$, qu'on lit de droite à gauche, tel que $L^{r}\left(Z_{d}\right)=\left\{w_{n} \ldots w_{1} \in\left(A_{E} \times\right.\right.$ $\left.\left.A_{S}\right)^{*} / w_{1} \ldots w_{n} \in L\left(Z_{d}\right)\right\}=L(\mathcal{T})$. Les transducteurs agissant de droite à gauche ont déjà été utilisés dans [BDT], pour représenter la multiplication par un nombre entier en base $p$. Lorsqu'une fonction $f$ du tore peut être représentée en base $p$ par $Z_{g}$ ou $Z_{d}$, on dit qu'elle est transductorielle (à droite ou à gauche respectivement).

Soit $x=\sum_{i=1}^{l} \frac{b_{i}}{p^{i}} \in \mathbb{T}, b_{i} \in A_{p}$. L'algorithme de multiplication par un entier, $k \in \mathbb{N}$, agit sur le mot $w=b_{1} \ldots b_{l}$, de droite à gauche, de la façon suivante : si $[k x]_{1}=\sum_{i=1}^{l} \frac{b_{i}^{\prime}}{p^{i}}$ alors $b_{i}^{\prime}=\left[k b_{i}+c_{i+1}\right]_{p} \in A_{p}$, la retenue $c_{i}=$ $\left\lfloor\frac{k b_{i}+c_{i+1}}{p}\right\rfloor$ et $c_{l+1}=0(\lfloor\cdot\rfloor$ est la partie entière inférieure). Un calcul simple montre que $c_{i} \in A_{k}$. Dans [BDT] on décrit un transducteur qui réalise la 
multiplication par $k$ en base $p$ suivant l'algorithme ci-dessus. Les états du transducteur correspondent aux retenues et un arc entre deux retenues $c$ et $b$ est étiqueté $\operatorname{par}(a, v) \in A_{p} \times A_{p}$ si et seulement si $k a+c=b p+v$.

Soit $a \in A_{p}$, on note $l_{a, p} \in A_{k}$ et $f_{a, p} \in A_{p}$ les entiers tels que $k a=$ $l_{a, p} p+f_{a, p}$. Lorsqu'il n'y a pas ambiguïté par rapport à la base on écrit simplement $l_{a}$ et $f_{a}$.

Le lemme suivant précise certains propriétés de l'algorithme de la multiplication.

Lemme 2.4. Soient $p, k \in \mathbb{N}$.

(i) Soit $a \in A_{p^{r}}, r \in \mathbb{N}$. Alors il existe $\left(b_{i}\right)_{i=1}^{r} \subseteq A_{p}$ tel que $k a=l_{a, p^{r}} p^{r}+$ $\sum_{i=1}^{r} b_{i} p^{r-i}$

(ii) Si $k$ divise $p(k / p)$, quel que soit $a \in A_{p}, f_{a, p}+k-1 \leq p-1$.

(iii) Si $k<p$ et $k$ ne divise aucun puissance de $p$ alors il existe $a_{0}, \ldots, a_{T-1} \in$ $A_{p}$ tels que pour tout $j \in A_{T}, f_{a_{j}, p}+l_{a_{[j+1]_{T}}}=p-1$ et $l_{a_{[j+1] T}} \in A_{k-1}$.

Preuve.

(i) C'est direct.

(ii) Supposons que $k$ divise $p$, donc $p=q k, q \in \mathbb{N}$, et $A_{p}=\{i q+j / i \in$ $\left.A_{k}, j \in A_{q}\right\}$. Si $a=i q+j \in A_{p}, k a=i k q+k j=i p+j k$ et $f_{a}=j k$. Finalement, $f_{a}+k-1=j k+k-1 \leq(q-1) k+k-1=p-1$, d'où le résultat.

(iii) Supposons que $k<p$ et $k$ ne divise aucun puissance de $p$. Donc, $\frac{1}{k}$ n'est pas un $p$-adique du tore, et son développement en base $=p$ est ultimement périodique avec partie périodique non nulle. Notons $\underline{x}=$ $\left(a_{0} \ldots a_{T}\right)^{\infty}$ la partie périodique. II est direct que $V_{p}(\underline{x}) \neq 0$. Soit $\underline{y}_{n}=$ $\left(a_{0} \ldots a_{T-1}\right)^{n} 0^{\infty} ; \underline{y}_{n}$ est le développement en base $p$ d'un point $y_{n}$ du tore. Choisissons $n$ assez grand, de façon que la suite des retenues obtenue en appliquant l'algorithme de la division par $k$ à $\underline{y}_{n}$ devient aussi périodique (à gauche). Notons $r_{i}$ la retenue associé à $a_{i}$. Alors $k a_{i}+r_{[i+1]_{T}}=$ $r_{i} p+s_{i}$. Comme $k \frac{1}{k}=1, s_{i}=0 \vee(p-1)$. Mais $y_{n}$ converge, par la gauche, vers $V_{p}(\underline{x})$, donc, forcement, $s_{i}=p-1$. En conséquence, $k a_{i}+r_{[i+1]_{T}}=r_{i} p+p-1=l_{a_{i}}=p+f_{a_{i}}+r_{[i+1]_{T}} ;$ comme $k<p$, $f_{a_{i}}+r_{[i+1]_{T}}=p-1$ et $l_{a_{i}}=r_{i} \in A_{k}$, d'où la première affirmation de (iii).

Pour finir supposons que $r_{i}=k-1$ pour un certain $i \in A_{T}$. Donc, $k a_{i}+r_{[i+1]_{T}}=(k-1) p+p-1=k p-1$, d'où $a_{i}=p-1$ et $r_{[i+1]_{T}}=k-1$. 
En itérant on déduit que $a_{i}=p-1$ et $r_{i}=k-1$ pour tout $i \in A_{T}$, ce qui est une contradiction. On a donc montré que $r_{i} \in A_{k-1}$ et par conséquent l'affirmation (iii).

Dans cet article, on ne considère que des automates cellulaires unilatéraux.

DÉfinition 2.5. Soit $A$ un alphabet fini. Un automate cellulaire (unilatéral) est une application $F: A^{\mathbb{N}} \rightarrow A^{\mathbb{N}}$ définie par :

$$
\forall \underline{x}=\left(x_{i}\right)_{i \in \mathbb{N}} \in A^{\mathbb{N}}, i \in \mathbb{N} \quad F(\underline{x})_{i}=f\left(x_{i} x_{i+1} \ldots x_{i+r}\right)
$$

où $f: A^{r+1} \rightarrow A$ est une application donnée. L'entier $r$ est appelé le rayon de l'automate cellulaire.

Soit $F: A_{p}^{\mathbb{N}} \rightarrow A_{p}^{\mathbb{N}}$ un automate cellulaire et $r$ son rayon. $F$ peut être vu comme un transducteur. L'ensemble d'états du transducteur est $A_{p}^{r}$ et un état $\left(a b_{1} \ldots b_{r-1}\right) \in A_{p}^{r}$ est connecté par un arc avec $\left(b_{1} \ldots b_{r-1} b\right) \in A_{p}^{r}$ pour tout $b \in A$. On étiquette ce graphe par $\left(a, F\left(a, b_{1}, \ldots, b_{r-1}, b\right)\right)$ sur l'arc qui relie les états $\left(a, b_{1}, \ldots, b_{r-1}\right)$ et $\left(b_{1}, \ldots, b_{r-1}, b\right)$. De cette façon pour chaque $\underline{x}=\left(x_{i}\right)_{i \in \mathbb{N}} \in A_{p}^{\mathbb{N}}$ il existe un unique état $q=\left(x_{1}, \ldots, x_{r}\right)$ du graphe depuis lequel on lit (de gauche à droite) $\underline{x}$. On lui associe les couplages $Z_{d}$ et $Z_{g}$ définis ci-dessus pour les transducteurs. On dit que $f: \mathbb{T} \rightarrow \mathbb{T}$ est réalisable par automate cellulaire en base $p$ si $Z_{d}$ ou $Z_{g}$ représente $f$ (on garde la notation pour indiquer de gauche à droite et l'inverse). Dans ce cas $Z_{g}$ est simplement le graphe d'une application.

\section{Résultats.}

Rappelons d'abord un lemme classique qui va nous servir immédiatement.

Lemme 3.1. Soit $f: \mathbb{T} \rightarrow \mathbb{T}$ une fonction continue du tore. II existe une fonction $g:[0,1] \rightarrow \mathbb{R}$ continue telle que $\forall x \in \mathbb{T}, f(x)=[g(x)]_{1}$, $g(0)=f(0)$ et $(g(1)-g(0)) \in \mathbb{Z}$.

Le résultat suivant, qui est une description complète du commutant topologique des multiplications entières $(\bmod 1)$ sur le tore, est connu depuis longtemps de H. Furstenberg et B. Weiss, et affirmé sous une forme un peu plus faible dans [JR]. Nous en donnons ici une preuve simple. 
Proposition 3.2. Si la fonction $f: \mathbb{T} \rightarrow \mathbb{T}$ est continue et commute avec la multiplication par $p \in \mathbb{N}(\bmod 1)$ alors il existe $u \in \mathbb{Z}, m \in A_{p}$ tels que $f(x)=\left[u x+\frac{m}{p-1}\right]_{1}$.

Preuve. On obtient le résultat en résolvant une équation de récurrence relative à la fonction $g$ du lemme précédent.

L'hypothèse de commutation se traduit, indépendamment de la continuité, par l'égalité suivante :

$$
\left(f(x)-p^{l} f\left(\frac{k+x}{p^{l}}\right)\right) \in \mathbb{Z}, \forall l \in \mathbb{N}, k \in\left\{0, \ldots, p^{l}-1\right\}, x \in \mathbb{T} .
$$

En fait (1) est aussi vrai pour $x=1$, en posant $f(0)=f(1)$.

D'après la proposition 2.1 et le lemme 3.1 , il existe $g:[0,1] \rightarrow \mathbb{R}$ telle que pour tout $x \in[0,1], f(x)=[g(x)]_{1}$. II s'ensuit que

$$
\begin{aligned}
C(k, l, x)= & \left(g(x)-p^{l} g\left(\frac{k+x}{p^{l}}\right)\right) \in \mathbb{Z}, \\
& \forall l \in \mathbb{N}, \forall k \in\left\{0, \ldots, p^{l}-1\right\}, x \in[0,1] .
\end{aligned}
$$

Mais toute fonction à valeurs entières continue sur l'intervalle est constante, d'où $C(k, l, x)=C(k, l)$. Calculons ces constantes. Si on évalue (2) aux points $x=0$ et $x=1$ on obtient,

$$
C(k, l)=\left(g(0)-p^{l} g\left(\frac{k}{p^{l}}\right)\right) \quad \text { et } \quad C(k, l)=\left(g(1)-p^{l} g\left(\frac{k+1}{p^{l}}\right)\right)
$$

En conséquence, si $k \in\left\{0, \ldots, p^{l}-2\right\}, C(k+1, l)-C(k, l)=g(0)-g(1)$, qui est donc entier, et $C(0, l)=\left(1-p^{l}\right) g(0)$, d'où

$$
C(k, l)=k(g(0)-g(1))+g(0)\left(1-p^{l}\right), \quad \forall k \in\left\{0, \ldots, p^{l}-1\right\}
$$

et

$$
\begin{aligned}
g\left(\frac{k}{p^{l}}\right) & =\frac{g(0)}{p^{l}}-\frac{C(k, l)}{p^{l}} \\
& =\frac{g(0)}{p^{l}}-\frac{k(g(0)-g(1))+g(0)\left(1-p^{l}\right)}{p^{l}} \\
& =\frac{k}{p^{l}}(g(1)-g(0))+g(0)
\end{aligned}
$$


Donc pour tous les p-adiques de $[0,1], g(x)=x(g(1)-g(0))+g(0)$; par continuité c'est aussi valable pour tout l'intervalle. Mais $g(1)-g(0)=u \in \mathbb{Z}$ et $g(0)=f(0)=v \in \mathbb{T}$, d'où $f(x)=[u x+v]_{1}$.

Pour finir il suffit de calculer $f(0)$. D'après (3.2), et en considérant $l=1$, $k=0$ et $x=0$, on obtient que $p f(0)-f(0)=(p-1) f(0)=m \in \mathbb{Z}$. Mais $m \in A_{p}$, d'où le résultat.

THÉORÈme 3.3. Si la fonction $f: \mathbb{T} \rightarrow \mathbb{T}$ a une représentation en base $p \in \mathbb{N}$ alors il existe $u \in \mathbb{Z}, m \in A_{p}$ tels que $f(x)=\left[u x+\frac{m}{p-1}\right]_{1}$.

Preuve. Elle s'obtient en enchaînant les Propositions 2.2 et 3.2.

En particulier le théorème 3.2 montre que les rotations irrationnelles du tore ne sont pas représentables en base $p$.

On a montré dans la deuxième partie de l'article qu'avec des transducteurs agissant de droite à gauche on pouvait représenter les fonctions $f(x)=[u x]_{1}, u \in \mathbb{N}$. En fait, avec une construction analogue on peut prouver le corollaire suivante.

Corollaire 3.4 Soit $p \in \mathbb{N}$. Toutes les fonctions $f(x)=\left[u x+\frac{m}{p-1}\right]_{1}$, $u \in \mathbb{Z}, m \in A_{p}$, sont transductorielles en base $p$ à gauche.

Preuve. Il suffit de modifier le transducteur de multiplication par $u$ [BDT], décrit dans la deuxième partie, de façon qu'il additionne au produit le nombre $\frac{m}{p-1}$, dont le développement est $m^{\infty}$.

Un calcul simple montre que l'ensemble des retenues peut alors devenir $A_{k+1}$. Alors un état (une retenue) $c_{1}$ est lié à un autre, $c_{2}$, par un arc d'étiquette $(a, b) \in A_{p} \times A_{p}$, si et seulement si $k a+c_{1}+\sigma=c_{2} p+b$. La preuve découle des arguments de [BDT].

Maintenant on caractérise les fonctions du tore qui sont représentables par automate cellulaire. Pour les automates cellulaires on considère seulement la transduction à droite; le cas à gauche est une conséquence de l'interprétation comme transducteur.

Corollaire 3.5 Une fonction $f: \mathbb{T} \rightarrow \mathbb{T}$ est réalisable par un automate cellulaire en base $p$ à droite si et seulement si $f(x)=[u x]_{1}$, où $u$ est un entier qui divise une puissance positive de $p$.

Preuve. Soient $p \in \mathbb{N}$ une base fixe et $F$ un automate cellulaire de rayon $r$ qui réalise $f$ à droite. D'après le théorème 3.3 il existe $u \in \mathbb{Z}$ et $\sigma \in A_{p}$ tels que $f(x)=\left[u x+\frac{m}{p-1}\right]_{1}$. 
Supposons $r$ minimal : c'est donc qu'il existe $B=b_{1} \ldots b_{r} \in A_{p}^{r}$ et $b \in$ $A_{p} \backslash\{0\}$ tels que $F\left(b_{1} \ldots b_{r} b\right)=a \neq F\left(b_{1} \ldots b_{r}(b-1)\right)$. Comme $\underline{x}=B b 0^{\infty}$ et $\underline{y}=B(b-1)(p-1)^{\infty}$ représentent le même élément $x \in \mathbb{T}, F(\underline{x})$ et $F(\underline{y})$ représentent $f(x)$. Alors, forcément, $F(\underline{x})=a 0^{\infty}$ et $F(\underline{y})=(a-1)(p-1)^{\infty}$ ou inversement. En conséquence $f(0)=0=m$.

Pour finir on va calculer les valeurs de $u$ possibles pour la représentation en base $p$. On considère seulement les $u$ positifs, pour les négatifs le calcul est analogue.

Soit $u \in \mathbb{N}$ tel que $u / p$. D'après le lemme 2.4 (ii), pour tout $a \in A_{p}$, $f_{a}+u-1 \leq p-1$. Cela signifie que quel que soit $R \in A_{u}, f_{a}+R \leq p-1$, et donc la retenue associée à $u a+R$ est $l_{a}$ indépendamment de $R$. Ainsi l'algorithme de la multiplication par un entier est local. Cela nous permet de bien définir un automate cellulaire qui représente $[u x]_{1}$ en base $p$ :

$$
\forall x_{0}, x_{1} \in A_{p} \text { on pose } F\left(x_{0}, x_{1}\right)=\left[u x_{0}+l_{x_{1}}\right]_{p}=f_{x_{0}}+l_{x_{1}} .
$$

En général, si $u$ divise $p^{r}$, d'après le lemme 2.4 (i), l'automate cellulaire est :

$$
\forall x_{0}, x_{1}, \ldots, x_{r} \in A_{p}, F\left(x_{0}, x_{1}, \ldots, x_{r}\right)=\left[u x_{0}+l_{x_{1}, \ldots, x_{r}}\right]_{p}
$$

où $l_{x_{1}, \ldots, x_{r}}=l_{a, p^{r}}$ et $a=\sum_{i=1}^{r} x_{i} p^{r-i} \in A_{p^{r}}$.

Maintenant montrons que si $u$ ne divise aucun puissance de $p$ alors il n'existe pas d'automate cellulaire qui réalise la multiplication par $u$ en base $p$. D'après le lemme 2.4 (i), et de façon analogue au cas précédent, on peut se restreindre au cas $u<p$.

Toujours par le lemme 2.4 (iii), il existe $a_{0}, \ldots, a_{n-1} \in A_{p}$ tels que pour tout $j \in A_{n}: f_{a_{j}}+l_{a_{[j+1]_{n}}}=p-1$ avec $l_{a_{j}} \in A_{u-1}$. In s'ensuit que la retenue associée à chaque $a_{j}$ dans l'algorithme de la multiplication par $u$ est soit $l_{a_{j}}$ soit $\left(l_{a_{j}}+1\right) ;\left(l_{a_{j}}+1\right)$ est attendu lorsque $u<p$ et ne divise pas $p$.

Quand $a_{j}$ reçoit comme retenue $l_{a_{[j+1]_{n}}}, u a_{j}+l_{a_{[j+1]_{n}}}=l_{a_{j}} p+f_{a_{j}}+$ $l_{a_{[j+1]_{n}}}=l_{a_{j}} p+p-1$ et il donne comme retenue $l_{a_{j}}$, d'autre part s'il reçoit $\left(l_{a_{[j+1]_{n}}}+1\right)$ il donne $l_{a_{j}}+1$.

Choisissons $b, b^{\prime} \in A_{p}$ tels que $u b^{\prime}-f_{b^{\prime}}=\left(l_{a_{1}}+1\right) p$ et $u b-f_{b}=l_{a_{1}} p$.

Soit $l \in \mathbb{N}$. Si $\underline{x}^{l}=\left(a_{0} \ldots . a_{n-1}\right)^{l} a_{0} b 0^{\infty}$ et $\underline{y}^{l}=\left(a_{0} \ldots . a_{n-1}\right)^{l} a_{0} b^{\prime} 0^{\infty}$, donc $\left[u \underline{x}^{l}\right]_{1}=((p-1) \ldots . .(p-1))^{l}(p-1) f_{b} 0^{\infty}$ et $[u \underline{y}]_{1}=(0 \ldots .0)^{l} 0 f_{b^{\prime}} 0^{\infty}$. En 
conséquence, s'il existe un automate cellulaire, $F$, qui réalise $f(x)=[u x]_{1}$ en base $p, F\left(\left(a_{0} \ldots a_{n-1}\right)^{\infty}\right)=(p-1)^{\infty}$ et $F\left(\left(a_{0} \ldots a_{n-1}\right)^{\infty}\right)=0^{\infty}$, ce qui est une contradiction.

Remarque 3.6. Le corollaire 3.5 montre aussi une façon de construire des transducteurs à droite qui réalisent la multiplication par $u$ en base $p$, quand $u$ divise une puissance de $p$.

Remarque 3.7. Soit $p=p_{0}^{n_{0}} \ldots p_{l}^{n_{l}} \in \mathbb{N}$, où les $p_{i}$ sont des nombres premiers. Si l'on note $F_{p_{i}}$ l'automate cellulaire qui réalise $f(x)=\left[p_{i} x\right]_{1}$, on obtient une factorisation du décalage, $\sigma=F_{p_{0}}^{n_{0}} \circ \ldots \circ F_{p_{l}}^{n_{l}}$, où les automates $F_{p_{i}}$ commutent deux à deux. Au surplus, si on considère maintenant leur action $\operatorname{sur} A_{p}^{\mathbb{Z}}$, ils sont bijectifs, et donc des automorphismes du shift.

\section{BIBLIOGRAPHIE}

[B] N. Bourbaki, Espaces vectoriels topologiques, Masson.

[BDT] F. Blanchard, J.M. Dumont, A. Thomas, Generic sequences, transducers and multiplication of normal numbers, Israel journal of Math 80, 257-287.

[BG] F. Botelho, M. Garzon, On dynamical properties of neural networks, Complex Systems 5 (1991), 401-413.

[JR] A. Johnson, D. J. Rudolph, Commuting endomorphisms of the circle, Ergodic Th. Dynam. Systems (1992).

[M] J.M. Muller, Some characterizations of functions computable in on-line arithmetic, Rapport LIP, 91-15.

François BlanchaRd et Bernard Host

Laboratoire de Mathématiques Discrètes

Case 930 - 163 avenue de Luminy

13288 Marseille Cedex 09, France

Alejandro MAASS

Departamento de Ingeniería Matemática

Universidad de Chile

Casilla 170/correo 3

Santiago, Chile 